\section{Eunice Nakamura'}

José Quirino dos Santos"
Departamento de Enfermagem em Saúde Coletiva. Escola de Enfermagem. Universidade de São Paulo. São Paulo, SP, Brasil

" Departamento de Psiquiatria. Universidade Federal de São Paulo. São Paulo, SP, Brasil

\author{
Correspondência | Correspondence: \\ Eunice Nakamura \\ Escola de Enfermagem da USP \\ Av. Dr. Enéas de Carvalho Aguiar, 419 \\ 05403-000 São Paulo, SP, Brasil \\ E-mail: eunice_nakamura@hotmail.com
}

Recebido: 11/5/2005 Revisado: 3/5/2006 Aprovado: 3/10/2006

\section{Depressão infantil: abordagem antropológica}

\section{Child depression: anthropological approach}

\section{RESUMO}

OBJETIVO: Apreender significados socioculturais da depressão infantil, a partir da noção médico-científica da doença.

MÉTODOS: Pesquisa qualitativa realizada em 2003, na região metropolitana de São Paulo, SP, Brasil. Utilizou-se a observação etnográfica e entrevistas em profundidade com oito médicos psiquiatras de um serviço público de saúde e nove familiares (pais ou responsáveis) de crianças com diagnóstico e em tratamento de depressão infantil. $\mathrm{Na}$ análise, buscou-se identificar categorias que permitissem isolar diferentes noções de doença expressas nos discursos desses grupos.

RESULTADOS: Foram identificadas diferentes noções da doença, de acordo com padrões culturais dos discursos. Para os psiquiatras, a noção de depressão infantil referiu-se a comportamentos infantis inadequados, ou "mau funcionamento", os quais devem ser ajustados pela intervenção médica. Para os familiares, significou "insatisfação" e "incômodo" diante da vida e "intolerância" de adultos frente a certos comportamentos infantis. Observou-se que os discursos dos médicos psiquiatras e dos familiares entrevistados mostraram diversidade quanto aos seus conteúdos, conceitos e categorias, segundo lógicas próprias de compreensão e de explicação da depressão infantil.

CONCLUSÕES: Diante dos resultados obtidos, o fenômeno da depressão infantil passa a ser analisado não como evento determinado pelo conhecimento médicocientífico, mas como um processo dinâmico de "reinvenção criadora" de categorias e conceitos fundamentais desse discurso. Concluiu-se, portanto, que a depressão infantil apresentou-se como doença diferenciada, na forma de uma categoria ampla, capaz de integrar diferentes conotações e contextos sob um mesmo termo.

DESCRITORES: Antropologia cultural. Depressão. Pesquisa qualitativa. Saúde mental. Bem-estar da criança.

\section{ABSTRACT}

OBJECTIVE: To understand the sociocultural meanings of childhood depression, from the medical-scientific concept of the disease.

METHODS: This was a qualitative study carried out in the metropolitan region of São Paulo, State of São Paulo, Brazil, in 2003. It consisted of ethnographic observation and in-depth interviews with eight psychiatrists from a public health service and nine relatives (parents or guardians) of children who had been diagnosed with and were being treated for childhood depression. The analysis sought to identify categories that would make it possible to isolate different notions of the disease, as expressed in the discourse of these groups.

RESULTS: Different notions of the disease were identified, in accordance with the 
cultural patterns of the discourse. For the psychiatrists, the concept of childhood depression was related to inappropriate child behavior, or "bad functioning", which then had to be adjusted by medical intervention. For the relatives, childhood depression meant "dissatisfaction" and "discomfort" with life and "intolerance" of adults to child behavior. It was seen that the discourse of the psychiatrists and relatives interviewed showed great diversity of subjects, concepts and categories, according to the logic of their particular understandings and explanations for childhood depression.

CONCLUSIONS: In the light of the results obtained, the phenomenon of childhood depression can be analyzed not as an event determined by medical-scientific knowledge, but as a dynamic process of "creative reinvention" of categories and concepts that are fundamental to this discourse. It is therefore concluded that childhood depression presents as a differentiated disease, in the form of a broad category that is capable of integrating different connotations and contexts under the same term.

\section{KEYWORDS: Anthropology, cultural. Depression. Qualitative research.} Mental health. Child welfare.

\section{INTRODUÇÃO}

Nos últimos anos, os chamados transtornos afetivos, dentre eles a depressão, adquiriram maior destaque no meio científico e passaram também a atrair a atenção da população. A depressão, especificamente, tem se mostrado mais evidente desde a década de 1970, quando aumentou o interesse no campo da investigação e no meio acadêmico e reconheceu-se a presença significativa desse distúrbio entre crianças e adolescentes. Desde então, passou-se a discutir a identidade da depressão infantil como forma clínica independente daquela encontrada em adultos. A noção médica da doença afirmou-se, assim, concomitantemente à consolidação da psiquiatria infantil como especialidade desvinculada da psiquiatria do adulto e da pediatria. ${ }^{1}$

Ao lado de um maior número de informações disponíveis sobre a depressão infantil e suas conseqüências individuais e sociais, tem sido crescente a preocupação com a doença. Na década de 1990, foram divulgados relatórios pela Organização Mundial da Saúde acerca da saúde mental e conseqüências dos problemas mentais à população. Segundo esses relatórios, houve aumento da depressão na população mundial, atingindo, na década de 1990, cerca de 330 milhões de pessoas, principalmente as mulheres. Entre elas, a incidência da doença é duas vezes maior do que entre os homens. Os relatórios mostram ainda que em 2020 a depressão será a segunda maior causa de doença, perdendo apenas para as doenças do coração, sendo alto o custo de seu tratamento, estimado em 44 bilhões de dólares ao ano para os Estados Unidos.

Embora o aumento da depressão seja mais evidente em mulheres, o seu aparecimento na população mas- culina adulta não está descartado, assim como a sua ocorrência na população jovem, ou seja, em crianças e adolescentes.

A depressão infantil é identificada pelo discurso médico-científico dominante como doença grave, especialmente pela incapacitação social associada. ${ }^{6,15}$ Ela vem-se disseminando rapidamente na população por intermédio da imprensa, o que nem sempre ocorre numa associação restrita à noção de doença. Verifica-se no geral que esta noção é simplificada, permitindo que o termo seja incorporado indistintamente pela população.

No presente estudo, o processo de disseminação do conceito médico-científico de depressão infantil é compreendido a partir de duas abordagens, que se complementam na perspectiva crítica da antropologia médica. A primeira delas, a da especificidade das representações da doença, se expressa em noções e significados particulares. ${ }^{7}$ A outra, é sobre os mecanismos socioculturais que explicam uma impossibilidade de que a noção médico-científica seja traduzida à lógica de outros atores sociais. ${ }^{2,5,13}$

Apesar da existência de ampla bibliografia sobre o tema, observa-se em estudos que relacionam a depressão a questões culturais uma predominância de pesquisas na área da psiquiatria transcultural. Autores como Lecrubier ${ }^{10}$ e Pate ${ }^{18}$ comparam a distribuição da depressão infantil em diferentes populações, a partir da variação transcultural observada no reconhecimento, diagnóstico e tratamento da doença, ressaltando a influência da cultura em estudos epidemiológicos da depressão.

$\mathrm{Na}$ área da antropologia médica, considerada uma 
área privilegiada para os estudos sobre doenças, destacam-se estudos que têm contribuído para a abordagem crítica e mais aprofundada do tema da depressão, nos quais são analisados conceitos e noções produzidas pelo discurso biomédico analisados. ${ }^{5,8,9} \mathrm{~A}$ antropologia médica propõe uma abordagem mais ampla e também crítica da relação entre doença e cultura em termos das noções e práticas envolvidas nessa relação. ${ }^{19}$ Possibilita que nos estudos sobre doenças mentais, a cultura deixe de ser apenas uma área auxiliar da psicopatologia, passando a contribuir para que sejam revelados aspectos pouco conhecidos pelos profissionais de saúde, como os padrões de comportamento e as representações de doença da população. Especificamente em estudos sobre doenças mentais na infância, noções de doença aparecem vinculadas à maneira específica como as crianças e seus comportamentos são percebidos em diferentes sociedades. Isso permite que elas sejam classificadas, segundo parâmetros de normalidade e de anormalidade socialmente aceitos. ${ }^{17}$

Nesta perspectiva, devem ser considerados os idiomas culturais para expressar o sofrimento e a aflição. Da mesma maneira, é preciso reconhecer que a psiquiatria integra determinada cultura e que a imposição de certas categorias não se ajusta adequadamente e nem abarca completamente as experiências de sofrimento que afligem as pessoas em outras sociedades. A apresentação clínica da depressão e da ansiedade não é portanto apenas uma função do contexto etnocultural do paciente, mas depende da estrutura do sistema de saúde, das categorias de diagnóstico, dos conceitos que são encontrados na mídia e no diálogo com a família, amigos e médicos. ${ }^{8}$

Os estudos mencionados evidenciam possíveis contribuições de um estudo antropológico sobre o tema da depressão infantil, demarcando-se as fronteiras entre as diferentes perspectivas adotadas por essa ciência e pela ciência médica, definindo, ao mesmo tempo, possíveis interfaces. É evidente a intenção de ampliar o recorte médico na escolha do próprio tema, operando ao mesmo tempo o seu deslocamento, na medida em que se busca compreender a depressão infantil como um fenômeno sociocultural. Assim, o objetivo do presente estudo foi apreender significados socioculturais da depressão infantil, a partir da noção médico-científica da doença.

\section{MÉTODOS}

Estudo qualitativo realizado com médicos psiquiatras de um serviço público especializado em psiquiatria da infância e da adolescência, localizado no Município de São Paulo, SP. Foram incluídos tam- bém familiares (pais ou responsáveis) de crianças (seis a 12 anos) com diagnóstico de depressão infantil, acompanhadas no mesmo serviço.

A pesquisa de campo foi norteada pela prática etnográfica, delimitando-se uma abordagem particular do tema da depressão infantil diferente de outros estudos, como os tradicionalmente conduzidos pela área médica.

No trabalho etnográfico, a realidade é abordada de maneira singular, num processo interpretativo do pesquisador, que observa as condições e o contexto no qual se coletam as informações. Posteriormente, essas informações são sistematizadas e articuladas para elaborar um conhecimento mais amplo e profundo sobre essa realidade, revelando-se experiências concretas e não generalizações amplas. ${ }^{3,12}$

A pesquisa teve como principal objeto os discursos sobre depressão infantil elaborados por diferentes atores sociais, de acordo com opiniões, sentimentos, atitudes e diferentes visões de mundo, associadas à experiência com o fenômeno - questões fundamentais nas pesquisas em antropologia médica. ${ }^{20}$

Tomou-se como ponto de partida a noção de depressão infantil como doença elaborada no discurso médico-científico, para compreender as transformações pelas quais passa a noção nos discursos de médicos psiquiatras e de familiares (pais ou responsáveis) de crianças deprimidas.

Para identificação e inclusão das crianças e seus familiares, foram estabelecidos vários critérios, explanados a seguir.

a) Diagnóstico de depressão: Foram considerados apenas casos de depressão maior, diagnosticados pelos médicos psiquiatras, segundo os critérios da quarta edição do Manual Diagnóstico e Estatístico de Transtornos Mentais (DSM-IV) e da Classificação Estatística Internacional de Doenças e Problemas Relacionados à Saúde (CID-10), em acompanhamento no serviço.

b) Faixa etária: crianças entre seis e 12 anos: Incluíram-se apenas os pacientes situados na faixa etária de seis a 12 anos, desconsiderando-se crianças que se encontravam abaixo dessa faixa e os adolescentes. As primeiras porque se encontram em uma fase ainda restrita dos laços sociais e os últimos porque vivenciam situações consideradas mais propícias para o desenvolvimento da depressão. ${ }^{4}$ Foram identificadas nove crianças, cujos familiares (pais ou responsáveis) foram contatados e convidados a participar das entrevistas.

c) Local de moradia: Todas as crianças identifica- 
das e seus familiares residiam na periferia de São Paulo e de outros municípios da região metropolitana, situação freqüente entre os usuários dos serviços públicos de saúde. O grupo de familiares foi constituído como um grupo sociocultural homogêneo por partilharem a experiência de lidar com a depressão infantil de suas crianças.

Todos os médicos psiquiatras envolvidos no atendimento de crianças deprimidas foram convidados a participar da pesquisa. Privilegiou-se o grupo de profissionais da área médica, numericamente maior e hegemônico, considerando-se também o fato de que são os principais agentes difusores do conhecimento científico, sendo considerados informantes-chave para compreender aspectos relevantes do discurso biomédico. $^{2}$

Foram conduzidas entrevistas com oito médicos psiquiatras e familiares das nove crianças com diagnóstico de depressão.

As entrevistas com os médicos psiquiatras foram realizadas no serviço de saúde ou em seus consultórios. Buscou-se conhecer seu discurso sobre a depressão infantil em termos de noção da doença, da definição de uma criança doente - a partir dos sintomas, encaminhamento, diagnóstico - e de percepções do comportamento familiar diante do diagnóstico.

As entrevistas com os familiares, em seus domicílios foram divididas em duas etapas. Na primeira, procurou-se apreender o que os familiares sabiam sobre depressão infantil e como reagiam diante do fenômeno, tanto com relação ao diagnóstico quanto ao tratamento realizado com as crianças. Na segunda, enfatizou-se a percepção dos comportamentos infantis em função da relação estabelecida pelas crianças com os amigos e com sua própria família.

O quadro das diferentes realidades dos entrevistados foi complementado pela observação dos contextos que compõem cada quadro particular, nas seguintes situações:

- acompanhamento de reuniões para discussão de casos de depressão infantil, reuniões com as famílias e outros eventos realizados no serviço de saúde, para a apreensão de procedimentos específicos à lógica médica no processo de formulação do diagnóstico de depressão infantil;

- visitas domiciliares, para conhecimento mais aprofundado da realidade familiar, dos locais de moradia e dos comportamentos infantis e isento, na medida do possível, das influências exercidas sobre a opinião da população, pelo serviço de saúde e pelos profissionais que ali atuam.
Os fatos observados, anotados pelo pesquisador, foram analisados complementarmente aos depoimentos. O material gravado e transcrito foi classificado com base em extratos de narrativas, frases repetitivas e não usuais identificadas nos depoimentos, analisados a partir da identificação de temas específicos considerados relevantes à compreensão dos significados do objeto estudado. ${ }^{16} \mathrm{O}$ material assim fragmentado foi sistematizado em categorias classificatórias, consideradas ferramentas conceituais importantes "para isolar noções abstratas e encadeá-las em proposições", ${ }^{11}$ associadas aos comportamentos infantis, à doença e ao tratamento.

As entrevistas analisadas, complementadas pelas observações de contextos particulares, mostraram-se suficientes para configurar relatos expressivos de experiências, opiniões e práticas particulares.

A análise qualitativa, em especial como empregada na pesquisa antropológica, “(...) consiste essencialmente em empregar atos, fatos, falas e interpretações para formar um modelo lógico que seja explicativo dessa realidade, na maioria das vezes inacessível aos indivíduos". ${ }^{14}$

Nesse sentido, buscou-se explicitar, na análise do conjunto de informações que expressam as experiências particulares dos entrevistados, os significados e noções de depressão infantil nos diferentes discursos. A partir do trabalho interpretativo e explicativo do antropólogo, revelaram-se as lógicas internas e as possíveis relações estabelecidas entre essas formas díspares de expressar realidades tão diversas.

Todas as entrevistas e observações foram realizadas mediante assinatura de termo de consentimento, garantindo-se o caráter voluntário e o anonimato dos participantes, atendendo às normas da Comissão de Ética para Análise de Projetos de Pesquisa (CAPPesq) do Hospital das Clínicas da Faculdade de Medicina da USP.

\section{RESULTADOS E DISCUSSÃO}

\section{Discurso médico sobre depressão infantil}

No discurso dos psiquiatras entrevistados, a noção de depressão infantil aparece como uma doença, não como um estado qualquer, sendo classificada a partir de parâmetros biomédicos bem definidos. Trata-se de uma patologia explicada principalmente por fatores genéticos ou orgânicos e psicossociais, embora a influência de fatores externos não esteja totalmente descartada.

“(...) quando você fala de depressão em psiquiatria, 
teoricamente você está falando de uma doença (...) Então, você está falando de uma alteração de humor, com tendência à tristeza e à falta de prazer com as coisas, sem relação com outras doenças, fisicas inclusive, e sem relação com eventos precipitantes". (Profissional n. 2)

Os entrevistados avaliam e qualificam determinados comportamentos infantis para a composição do quadro de diagnóstico, a partir de informações trazidas pela família, o que pode limitar a atuação profissional diante do filtro imposto pela percepção leiga, familiar ou de outras pessoas. Isso implica julgar e avaliar os comportamentos infantis a partir de diferentes formas de percepção ou de acordo com graus de tolerância distintos.

“(...) quanto mais jovem ela [a criança] for, mais ela vai estar atrelada ou dependente do familiar que a está trazendo (...) na nossa área, a gente precisa muito ter uma boa noção de desenvolvimento normal, do que é uma criança normal, porque às vezes a família transforma em sintoma alguma coisa que ela considera anormal, mas que não é necessariamente. Às vezes, é só uma reação normal da criança a uma situação ambiental anormal". (Profissional n. 4)

Em geral, isso se deve ao fato de que a população, conforme os entrevistados, confunde determinados comportamentos infantis.

"Os pais podem considerar que aquilo é uma preguiça, a criança está com preguiça ou ela está birrenta. Acham que faz parte do processo de desenvolvimento, é uma fase, que ela não está doente, está passando por uma fase". (Profissional n. 5)

De acordo com os médicos psiquiatras, as famílias percebem e classificam determinados comportamentos infantis segundo seus próprios referenciais. A maioria delas manifesta tanto tolerância frente ao problema quanto impossibilidade de gerenciá-lo, o que é determinante em sua decisão de recorrer ou não à ajuda profissional.

"A criança, ela é levada porque ela aborrece ou porque ela não corresponde às expectativas". (Profissional n. 2)

É por esse motivo que os profissionais percebem, no geral, satisfação e alívio das famílias com o diagnóstico proposto, uma vez que o diagnóstico explica parte de seus problemas e de suas angústias incompreendidas.

Situações de resistência ao diagnóstico e ao tratamento, no entanto, são freqüentes. Na visão dos pro- fissionais, em especial quando a família é pouco informada, não sabe o que é depressão infantil, ou não aceita que depressão possa ocorrer em crianças.

"De um modo geral, elas [as famílias] não conseguem conceber como uma criança pode ficar deprimida. A criança supostamente tem que brincar, ficar feliz, não tem preocupações, não tem problemas, não tem compromisso maior além do estudo (...) Então, o conceito do adulto é que depressão é uma doença de adultos, não de crianças". (Profissional n. 5)

Com relação às formas de tratamento, os profissionais são unânimes quanto à necessidade de utilização de medicamentos e de os resultados mais imediatos serem reconhecidos. Embora o acompanhamento terapêutico também seja recomendado numa abordagem múltipla, de maneira diferente do que ocorre com os medicamentos, há controvérsias quanto à sua adoção. Isso em função de problemas relacionados à duração, à existência de profissionais e aos custos, principalmente por se tratar de um serviço público.

A intervenção profissional deve diminuir o sofrimento infantil, aumentar a "capacidade adaptativa" e proporcionar o ajuste dessas crianças. O uso de medicamentos parece atender de forma mais imediata a essas necessidades, mantendo-se como prática eficaz pela rapidez dos resultados observados, condizentes com o discurso médico-científico, pois para os médicos o importante é diagnosticar e curar.

Ao rotularem determinados comportamentos infantis como doença e prescreverem o tratamento, os profissionais explicitaram uma noção de depressão infantil que remete à idéia de adaptação e de ajuste. Para eles, a depressão infantil está vinculada a um "mau funcionamento" das crianças, sobre o qual a lógica médicocientífica garante a possibilidade de intervenção.

\section{Noção popular das famílias sobre depressão infantil}

Fato importante nas experiências familiares com relação à depressão infantil, no dizer espontâneo dos familiares entrevistados, é a incompreensão sobre o que estava acontecendo com as crianças. Os entrevistados não tinham idéia e percepção claras sobre o problema, o que teria adiado a procura de ajuda profissional, a identificação da doença e também a aceitação de que uma criança pudesse ficar deprimida. Os traços que apontavam para a doença só foram percebidos quando passaram a compor um quadro de atitudes e comportamentos estranhos o suficiente para serem destacados dos demais e recorrentes a ponto de chamar a atenção dos adultos. 
"Ele era muito nervoso, muito irritado, então às vezes, a gente não sabia o que era isso (...) a gente foi levando em banho maria, quer dizer, passou para a gente despercebido". (Família n. 7)

Esses estados infantis poderiam passar despercebidos ou adquirir relevância, dependendo de quem os observasse, do que fosse observado e do grau de tolerância do observador, nessas situações. Muitas vezes, os familiares mostravam certa confusão sobre os diferentes estados infantis definidos pela utilização de termos como "manhoso", "birrento", "nervoso" ou "mal-educado".

\section{"A gente pensava que era manha (...) a gente não entendia”. (Família n. 7)}

Ao serem percebidos como diferentes, os comportamentos infantis e as próprias crianças passaram a ser incorporados aos problemas que, de fato, incomodavam os adultos. Estabelecia-se assim uma relação evidente entre o grau de tolerância ou de intolerância a esses comportamentos e as condições gerais de vida das famílias. Nos depoimentos dos entrevistados, dramas familiares, como separações, brigas, mortes, alcoolismo e questões socioeconômicas, em especial desemprego e baixos salários, foram mencionados como fatores que alteram a dinâmica familiar, podendo afetar as crianças.

"Eu sou separada, quando me separei do meu marido, ele [o menino] tinha três anos. Ele sempre foi muito ligado ao pai e a separação foi muito violenta, teve muita briga, teve muita coisa. Nós inclusive, eu e o meu ex-marido, achávamos que não ia afetálo, principalmente por ele só ter três anos. Pelo contrário, afetou e até demais". (Família n. 6)

Nessas situações, muitas vezes as crianças passaram do papel de vítimas ao principal foco desses problemas, agravando a insatisfação dos adultos com a situação socioeconômica, fazendo com que os familiares, confusos, não soubessem como enfrentar a situação. À medida que a situação escapava do controle ou da classificação de normal ou aceitável das famílias, o desconhecimento, a incompreensão e a dúvida davam lugar ao desespero, ao medo e até à revolta, causada pela sensação de impotência, de certo modo, diante da própria vida.

"Eu me sentia muito revoltada (...) Sabe, quando você fala assim 'não tem mais solução! Não existe mais jeito', era assim que eu pensava”. (Família n. 3)

Para os familiares entrevistadas, a percepção do problema e de sua gravidade não estava vinculada dire- tamente aos aspectos clínicos que definem a noção de depressão como doença, mas ao desconforto sociocultural por ele causado.

Nessa perspectiva, a noção de depressão infantil expressa no discurso dos familiares revelou insatisfação e desconforto em como lidar com determinadas incertezas da vida, remetendo a uma situação de falta de controle diante do problema.

A depressão infantil surgiu como uma das explicações possíveis aos dramas vividos por esses familiares. A identificação de um termo para designar a situação confusa e especial que caracteriza a novidade de uma criança diferente e estranha para a família faz cessar a infinidade de tentativas de compreensão do problema.

\section{Relação entre os diferentes discursos}

Em função de contextos específicos, a constatação das diferenças entre os discursos dos médicos e dos familiares das crianças permitiu que o fenômeno da depressão infantil fosse analisado não como evento determinado pelo conhecimento médico-científico, mas a partir desse conhecimento e mediante um "jogo de reinterpretações" (Boltanski²) na análise das representações da doença. Nesse processo dinâmico de criação e de reinvenção, ou de "reinvenção criadora", segundo o autor, as categorias e os conceitos fundamentais do discurso médico-científico sofrem alterações, para que o universo estranho e desconhecido da medicina e dos médicos adapte-se às necessidades de outros atores sociais.

Analisados em seus contextos específicos, os discursos dos médicos psiquiatras e dos familiares entrevistados evidenciaram diferença quanto aos seus conteúdos, conceitos e categorias. Esses discursos se relacionam mediante lógicas próprias de compreensão e de explicação do fenômeno, de acordo com a visão de mundo desses diferentes atores sociais.

Possíveis semelhanças entre esses discursos podem ser apontadas apenas num plano mais imediato de apreensão de como, no diálogo entre médicos psiquiatras e familiares entrevistadas, estabelece-se uma troca na qual circulam conceitos associados à noção médico-científica de depressão infantil.

A impossibilidade de uma simples assimilação dos discursos tornou-se evidente, pois percebeu-se a demarcação de diferenças e a impossibilidade de correspondência ou de tradução de uma lógica para outra. As representações sobre depressão infantil elaboradas pelos médicos psiquiatras e pelos familiares referiram-se a visões de mundo e experiências 
concretas diversas entre si. Da mesma maneira, suas noções particulares de depressão infantil refletiram o campo de variação dos significados atribuídos a certos termos.

A aparente utilização indiscriminada do termo depressão infantil não corresponde, portanto, à incorporação da mesma lógica que fundamenta a noção de doença elaborada no discurso médico-científico, pelos diferentes grupos da sociedade. Segundo Lévi-Strauss, ${ }^{13}$ é no plano das estruturas inconscientes que uma forma aparentemente simples de repetição contrapõe-se à complexidade dos diferentes discursos, revelando lógicas específicas estruturadas a partir de um mecanismo considerado fundamental: a bricolage. Aqui, esse mecanismo é ressaltado como um dos principais elementos operadores da cultura na percepção e explicação do fenômeno da depressão infantil por diferentes atores sociais. A bricolage permite compreender como as diferentes noções de depressão infantil manifestamse num contexto marcado pelo esforço de homogeneizar o discurso médico-científico.

A partir dos elementos introduzidos pela cultura, mediante o discurso científico - informações, conceitos e categorias relacionadas à doença -, estabelecem-se arranjos considerados mais apropriados para cada grupo de atores sociais. Assim, as pessoas organizam suas vidas de acordo com a sua cultura, respondendo à necessidade cultural de ordenar o pen- samento diante dos acontecimentos da realidade.

\section{CONSIDERAÇÕES FINAIS}

A depressão infantil configura-se como uma categorização possível para aspectos diversos da realidade, permitindo classificar diferentes ordens de problemas: as disfunções orgânicas, socioeconômicas e culturais. Correspondem, respectivamente, a noções relacionadas ao mau funcionamento das crianças e de insatisfação, intolerância e infelicidade dos adultos ante a vida e seus acontecimentos. ${ }^{*}$ Tal como ocorre com relação às categorias de doença, em diferentes culturas, que permitem classificar os males e os infortúnios, é necessário organizar o que parecia desorganizado pelo restabelecimento da ordem.

A depressão infantil pode ser considerada uma doença diferenciada, porque opera culturalmente de forma diversa, se comparada a outras doenças, inclusive mentais. Revela-se como uma possibilidade de categorização inédita e improvisada de realidades diversas, tornando-se fundamentalmente necessária para identificar e organizar determinados comportamentos infantis e outros aspectos socioculturais emergentes. Atua, portanto, como um termo-chave operando várias noções com significados particulares, que se equivalem no modo como, graças a mecanismos de várias ordens, é possível explicar e, ao mesmo tempo, intervir sobre diferentes problemas da realidade.

\section{REFERÊNCIAS}

1. Assumpção Junior FB, Kuczynski E. Transtornos do humor. In: Assumpção Junior FB, Kuczynski E, organizadores. Tratado de psiquiatria da infância e da adolescência. São Paulo: Atheneu; 2003. p. 307-20.

2. Boltanski L. As classes sociais e o corpo. $3^{\text {a }}$ ed. Rio de Janeiro: Graal; 1989. (Biblioteca de Saúde e Sociedade, 5).

3. Oliveira RC. O trabalho do antropólogo. Brasília (DF): Paralelo 15/São Paulo: Unesp; 1998.

4. Ekblad S. Helping children cope with urban environment stress in developing countries. In: Harpham T, Blue I, editors. Urbanization and mental health in developing countries. Aldershot: Avebury; 1995. p. 103-21.

5. Fernando S. Mental health, race \& culture. London: MacMillan Press; 1991.
6. Gill SK, Coffey BJ, Park KS. Depressão na infância e na adolescência: manifestações clínicas, patogênese e tratamento. In: Lafer B, Almeida OP, Fraguas Jr R, Miguel EC, editores. Depressão no ciclo da vida. Porto Alegre: Artmed; 2000. p. 232-45.

7. Herzlich C. A problemática da representação social e sua utilidade no campo da doença. Physis. $1991 ; 1(2): 23-36$.

8. Kirmayer LJ. Cultural variations in the clinical presentation of depression and anxiety: implications for diagnosis and treatment. J Clin Psychiatry. 2001;62(Suppl 13):22-8.

9. Kleinman A, Good B, editors. Culture and depression: studies in the anthropology and cross-cultural psychiatry of affect and disorder. Berkley: University of California Press; 1985. p. 216-43.

*Esta possibilidade inédita de categorização é complementada pela análise das noções de depressão infantil do discurso médico-científico e da imprensa. (Nakamura E. Depressão na infância: uma abordagem antropológica [tese de doutorado]. São Paulo: USP; 2004) 
10. Lecrubier Y. Prescribing patterns for depression and anxiety worldwide. J Clin Psychiatry. 2001;62(Suppl 13):31-6.

11. Lévi-Strauss C. O cru e o cozido. São Paulo: Brasiliense; 1991.

12. Lévi-Strauss C. Antropologia estrutural dois. $4^{\text {a }}$ ed. Rio de Janeiro: Tempo Brasileiro; 1993. (Biblioteca Tempo Universitário, 45).

13. Lévi-Strauss C. O pensamento selvagem. $3^{\text {a }}$ ed. Campinas: Papirus; 2002.

14. Martin D, Andreoli SB, Quirino J, Nakamura E. Noção de significado nas pesquisas qualitativas em saúde: a contribuição da antropologia. Rev Saúde Pública. 2006;40:178-9.
15. Menezes PR, Nascimento AF. Epidemiologia da depressão nas diversas fases da vida. In: Lafer B, Almeida OP, Fraguas Jr R, Miguel EC, editores. Depressão no ciclo da vida. Porto Alegre: Artmed; 2000. p. 29-36.

16. Minayo MCS. O desafio do conhecimento: pesquisa qualitativa em saúde. São Paulo: Hucitec/Abrasco; 1992.

17. Nakamura E. Antropologia. In: Assumpção Junior F, Kuczynski E, organizadores. Tratado de psiquiatria da infância e da adolescência. São Paulo: Atheneu; 2003. p. 59-62.

18. Patel V. Cultural factors and international epidemiology. Br Med Bull. 2001;57:33-45.

19. Santos JQ. Adesão a tratamentos médicos. Psiquiatr Prat Med. 2000;33:14-6.

20. Sargent CF, Johnson TM, editors. Medical anthropology: contemporary theory and method. New York: Praeger Publishers; 1996.

Artigo baseado em tese de doutorado de E Nakamura, apresentada à Faculdade de Filosofia, Letras e Ciências Humanas da Universidade de São Paulo, em 2004. 